\title{
Heterogeneity of the Neural Composition of Cortical Regions as a Condition for a Wide Range Regulating Spontaneous Activity
}

\author{
Yulia S. Mednikova*, Anna V. Rogal \\ Institute of Higher Nervous Activity and Neurophysiology of RAS, Moscow, Russia \\ Email: *mednikova.yu.s@ihna.ru
}

How to cite this paper: Mednikova, Y.S. and Rogal, A.V. (2020) Heterogeneity of the Neural Composition of Cortical Regions as a Condition for a Wide Range Regulating Spontaneous Activity. Journal of Behavioral and Brain Science, 10, 220-236.

https://doi.org/10.4236/jbbs.2020.105014

Received: April 2, 2020

Accepted: May 22, 2020

Published: May 25, 2020

Copyright $\odot 2020$ by author(s) and Scientific Research Publishing Inc. This work is licensed under the Creative Commons Attribution International License (CC BY 4.0).

http://creativecommons.org/licenses/by/4.0/

(c) (i) Open Access

\begin{abstract}
On the slices of sensorimotor and frontal cortex in layer $\mathrm{V}$ of guinea pigs the diversity of neuronal spontaneous activity, the mechanism of its origin and functional specificity were studied. In both regions, neurons that did not have spontaneous activity predominated (39\% in the sensorimotor cortex and 32\% in the frontal cortex) over neurons with any other firing levels. The iontophoretic application of the excitatory transmitter, glutamate, caused activation spike reactions in all registered neurons; moreover, short-term activation reactions to glutamate had a significantly longer after-discharge in neurons of the frontal cortex (up to $2500 \mathrm{~ms}$ and more) compared to reactions of neurons of the sensorimotor region. This means that postexcitatory hyperpolarization in nerve cells of the frontal cortex is less expressed and, therefore, they have a lower density of $\mathrm{K}^{+}$channels on their membranes. With an increase in the level of spontaneous activity, $\mathrm{K}^{+}$membrane permeability decreases, which is confirmed by the appearance of a long activation reaction to acetylcholine (which blocks $\mathrm{K}^{+}$channels), exactly when spontaneous firing appears in "silent" neurons. Despite the fact that spontaneous activity is formed by glutamatergic excitation, its considerable diversity is associated with the structural and membrane characteristics of neurons, which determine the different degrees of EPSPs attenuation on the way of moving along dendrites. Acetylcholine regulates this process in different ways, in accordance with different states of $\mathrm{K}^{+}$membrane permeability. Therefore, the lower content of $\mathrm{K}^{+}$ channels on the membranes of neurons of the frontal cortex does not allow regulating spontaneous activity in the same range as occurs in neurons of the sensorimotor region. The presence of a high proportion of spontaneously inactive neurons in the cortex (higher in the sensorimotor cortex) suggests that cortical neurons are generally characterized by a high density of $\mathrm{K}^{+}$channels
\end{abstract}


and a significant increase in firing response to acetylcholine, while spontaneously active neurons cannot control the spontaneous activity in a wide range.

\section{Keywords}

Sensorimotor Cortex, Frontal Cortex, Spontaneous Activity, Glutamate, Post-Activation Aftereffect, Acetylcholine

\section{Introduction}

The diversity of cellular composition, even among the same type of neurons, is an obligatory characteristic of any brain formation [1]-[6] and varies when comparing homologous structures in the nervous system of different species animals [7] [8]. The heterogeneity of the cell composition was first detected among spinal cord motor neurons, which, varying in size of cell bodies, formed different spike sequences in response to standard excitation [2]. The morphological characteristics of neurons also differ in different lengths of dendrites and the degree of their branching [3] [4] [9]. These two parameters - cell body size and structure of dendritic tree are the morphological properties of neurons that determine the degree of the EPSPs amplitude attenuation when they move to the soma [10]. By blocking $\mathrm{K}^{+}$channels on the neuronal membrane, whose different densities are another source of cell diversity [11], the M-cholinergic reaction [12] [13] allows the synaptic flow formed in dendrites to transform into a somatic spike sequence [14].

In papers of many authors, it can be found that neurons of the same brain structure differ in parameters that determine the level of spontaneous activity and the degree of its variability. Thus, a more complex dendritic organization correlates with a lower frequency of spontaneous activity [9], low $\mathrm{K}^{+}$current density is observed in neurons with a high level of spontaneous firing [5], and neurons with high $\mathrm{K}^{+}$permeability are characterized by significant variability in the frequency of spontaneous activity [15]. High-frequency cortical neurons respond poorly to an increase in the rate of cholinergic reaction (blocking $\mathrm{K}^{+}$permeability) with increasing temperature, while in low-level cells under the same conditions, when the temperature approaches $36^{\circ} \mathrm{C}$, there is a sharp increase in the impulse response to acetylcholine [16]. These data indicate a high variability of spontaneous firing in low-activity neurons.

Since the formation of spontaneous activity is based on glutamatergic excitation, and its regulation occurs due to a change in the dendritic membrane properties under the influence of acetylcholine [14], both of these mediators, can provide the necessary variability in the response of the population as a whole if it is formed by neurons with heterogeneous functional possibilities to perform their own contributions. 
Based on these considerations, we conducted a comparative analysis of the functional peculiarities of neurons in two cortical regions: sensorimotor and frontal. We recorded the spontaneous activity of neurons of the layer V, the characteristics of the activation responses of nerve cells to the local application of glutamate and acetylcholine under stationary conditions and in the course of changing the spontaneous activity levels.

\section{Methods}

\subsection{Slice Preparation}

The experiments were carried out on the slices of the sensorimotor and frontal cortex of guinea pigs of both sex and 1.5 months old (200 - $250 \mathrm{~g})$. Animals were treated with observance of recommendation on ethics of work with animals offered by European Communities Council Direction (86/609 EEC) and experimental protocols approved by ethics committees of Institute of Higher Nervous Activity Russian Academy of Sciences. After quick decapitation of the animals by the guillotine and subsequent opening of the skull, the surface of the brain was irrigated with ice-cold aerated Ringer-Krebs solution. Slices of a sensorimotor or frontal cortex $500 \mu \mathrm{m}$ thick were prepared from a longitudinal block on a VSL vibrotome (World Precision Instruments, USA). One of the slices was placed in the experimental chamber, the rest stayed in the incubating one. The incubation conditions in both cells were the same. The incubating medium saturated with carbogen $\left(95 \% \mathrm{O}_{2}\right.$ and $\left.5 \% \mathrm{CO}_{2}\right)$ consisted of the following components, (mM): $124 \mathrm{NaCl} ; 5 \mathrm{KCl} ; 1.24 \mathrm{KH}_{2} \mathrm{PO}_{4} ; 1.3 \mathrm{MgSO}_{4} ; 2.4 \mathrm{CaCl}_{2} ; 26 \mathrm{NaHCO}_{3}$ and 10 glucose ( $\mathrm{pH}$ 7.4). It represents a composition of artificial serebrospinal fluid [13] [15]. The flow rate was $1.5-3 \mathrm{ml} / \mathrm{min}$. For half an hour after preparation, slices were incubated at room temperature. Then the temperature of the medium was gradually increased to $32^{\circ} \mathrm{C}-34^{\circ} \mathrm{C}$ and slices were incubated at this temperature for another 1.5 hours before the start of the experiment. Pre-heating of the incubating medium was carried out using a U1 thermostat (VEB, Germany); for fine temperature control, a thermostatic device based on a Peltier element (NPO "Biopribor", Russia) was used. The temperature in the experimental chamber was constantly measured by an electronic thermometer (STC "NIKAS", Russia). A standard temperature of $32^{\circ} \mathrm{C}-34^{\circ} \mathrm{C}$ was maintained throughout the experiment.

\subsection{Extracellular Recording and Transmitter Iontophoresis}

For extracellular registration of the impulse activity of neurons and iontophoretic injection of glutamate and acetylcholine to their bodies, three-channel glass microelectrodes with a total tip diameter of $7.4-8 \mu$ mwere used. The recording channel was filled with $3 \mathrm{M} \mathrm{NaCl}$ solution, the second and third channels contained 1M sodium glutamate (pH 7.5; Sigma Chemical Co., USA) and $2 \mathrm{M}$ acetylcholine chloride ( $\mathrm{pH}$ 4.0; Sigma Chemical Co., USA). Sometimes one of the phoretic channels was filled with $3 \mathrm{M} \mathrm{NaCl}$ solution to test the current effect of the applications of mediators. 


\subsection{Experimental Procedure}

A microelectrode containing recording and phoretic channels was placed at the level of the layer $\mathrm{V}$ of the cortex (1.5 - $1.6 \mathrm{~mm}$ from the pial surface) in order to search for the spike activity of neurons and local applications of mediators: glutamate and acetylcholine. Glutamate was injected by a current of $80 \mathrm{nA}$ (negative pole inside the electrode) and a duration of exposure of $0.5-1.5 \mathrm{~s}$; acetylcholine was applied with a current of $70 \mathrm{nA}$ (positive pole inside the electrode) always for $4.5 \mathrm{~s}$. A holding current of $3-5 \mathrm{nA}$ in opposite directions was set in both the phoretic channels throughout intervals between iontophoretic ejections. The experimental procedure performed on the slices of sensorimotor and frontal cortex was the same: after detecting the impulse activity of a neuron, spontaneous firing were recorded for 1 - 3 minutes, followed by 2 - 3 applications of glutamate and, as a rule, a single application of acetylcholine with long-lasting periods of post-ejections registration. In spontaneously inactive neurons, if registration conditions allowed, the testing procedure was repeated for an hour every 10 minutes in order to detect the possible occurrence of regular spike activity.

\subsection{Recording Equipment and Data Analysis}

The impulse activity of neurons after amplification (DAM 80, World Precision Instruments, USA) was introduced into an Intel (R) Core (TM) 2Duo CPU computer for storage, reproduction, and signal processing. Impulse activity parameters were analyzed using the Power-Graph computer program, version 3.3, Russia. The level of spontaneous activity and response parameters to the iontophoretic applications of mediators was determined: latent period; activation aftereffect or duration of the reaction; the intensity of the reaction. The frequency of spontaneous activity of neurons of the sensorimotor and frontal cortex was determined as the average for the 30 second period preceding the application of mediators. For the latent period, the interval from the beginning of phoresis to the development of the effect, which was half the maximum, was taken. The duration of the after-discharge was determined as the interval from the moment of termination of the phoresis to half weakening of the response (for application of glutamate) or for a period limited by the spike frequency, which was half of the maximum (for application of acetylcholine). To estimate the intensity of the reaction to the application of transmitters, the maximum moving average frequency was calculated from three consecutive $200 \mathrm{~ms}$ bins (for glutamate) or 500 $\mathrm{ms}$ bins (for acetylcholine) during the response period and compared with the analogous value calculated in the background. The necessary statistical analysis of the differences in the calculated parameters was carried out using the homogeneity criterion $\chi^{2}$.

\section{Results}

\subsection{Spontaneous Activity and Its Regulation}

In two series of experiments conducted on the slices of sensorimotor and frontal 
cortex of guinea pigs, activity of 314 neurons was recorded at layer V level (160 in the sensorimotor cortex and 154 in the frontal cortex). The rate of spontaneous activity in different neurons ranged from 0 to $25 \mathrm{imp} / \mathrm{s}$ and from 0 to 30 $\mathrm{imp} / \mathrm{s}$ (correspondingly in the sensorimotor and frontal regions). Distributions of registered neurons in accordance with the level of spontaneous firing are presented in Figure 1. The parameter of spontaneous activity is a reliable indicator of a significant variety of cellular composition both in the sensorimotor and in the frontal regions of the cortex. In both zones, spontaneously inactive neurons predominated. The presence of such neurons was identified by the appearance of spike activity in response to glutamate applications by short current pulses. Of 160 neurons of the sensorimotor cortex, 63 (39\%) were inactive; of 154 cells of the frontal region - $50(32 \%)$. Despite the absence of significant differences in the distributions of neurons according to the level of spontaneous activity in both cortical regions, there is a slight displacement of frontal cortex neurons towards high-frequency activity: there are fewer spontaneously inactive neurons in the frontal cortex, neurons with a maximum spike frequency are also recorded there, and the proportion of high-frequency nerve cells (higher than 8 pulses/s) is $27 \%$ compared with $19 \%$ in the sensorimotor cortex (Figure 1).

Spontaneous activity is not only different in different neurons, it is the most variable parameter among the electrophysiological properties of nerve cells. The predominance of spontaneously inactive neurons in slices allows to trace the cause of such variability. In Figure 2, I on the example of a spontaneously inactive neuron of the frontal cortex, it is shown what is the initial reactions that

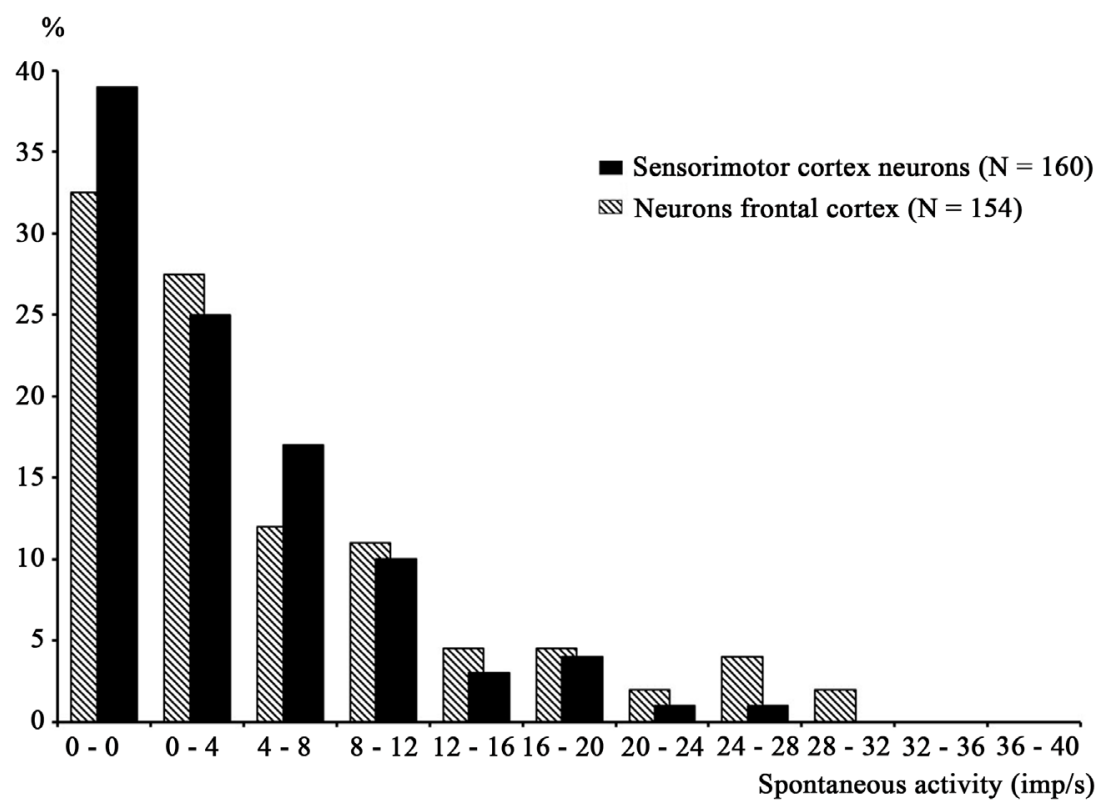

Figure 1. Distribution of neurons of the sensorimotor and frontal cortex according to the frequency of spontaneous activity. The abscissa shows the frequency of spontaneous activity (imp/s); The ordinate axis is the percentage of neurons that have a given level of spontaneous firing. At the top right is the number of registered neurons in the sensorimotor and frontal cortex. 
I - frontal cortex

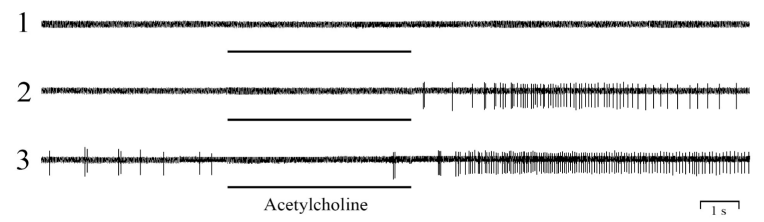

II - sensorimotor cortex

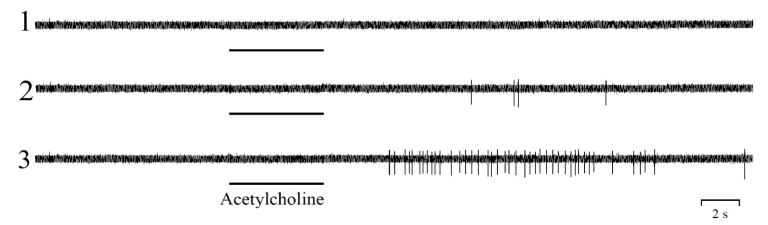

(A)
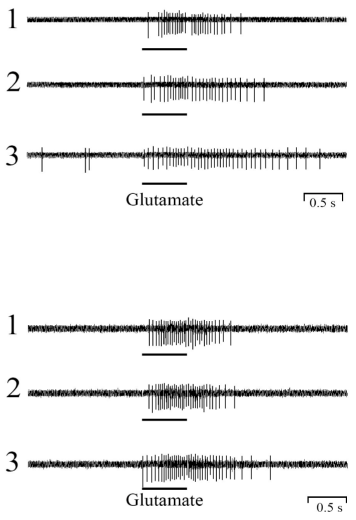

(B)

Figure 2. The formation of spontaneous activity in two spontaneously inactive neurons. I: neuron of the frontal cortex; II: neuron of the sensorimotor cortex. (A): reaction of neurons to the iontophoretic application of acetylcholine: phoretic current $70 \mathrm{nA}$ (positive pole inside the electrode); (B): reactions of neurons to the iontophoretic application of glutamate: phoretic current $80 \mathrm{nA}$ (negative pole inside the electrode); 1, 2, 3: three consecutive presentations of transmitters every 10 minutes. In each sample, glutamate testing was always performed before presentation of acetylcholine. The duration of the phoretic current of mediators is indicated by a bar under each recording.

arouse the appearance of spontaneous firing. The iontophoretic application of glutamate and acetylcholine to the neuron every 10 minutes allows to see that the appearance of spontaneous activity is preceded by the appearance of a long activation reaction to the application of acetylcholine (Figure 2(A), I, 2), which was not observed 10 minutes before (Figure 2(A), I, 1). After another $10 \mathrm{mi}$ nutes, in addition to the enhanced reaction to acetylcholine, regular spontaneous activity is recorded (Figure 2(A), I, 3). At the same time, whatever the level of spontaneous activity exists, the activation reaction to glutamate is reproduced stably in all three cases (Figure 2(B), I, 1 - 3). But in the course of spontaneous activity development the response to the iontophoretic application of glutamate acquires an increasingly long postexciting after-discharge, which is twice of its initial duration at the absence of spontaneous activity (Figure 2(B), I, 1 and 3). This means that: 1) the response to glutamate is stable and cannot be considered as a regulator of spontaneous activity; 2) the regulator of spontaneous activity is associated with the state of $\mathrm{K}^{+}$permeability, which is identified by the prolongation of the activation aftereffect; 3 ) spontaneous activity and reaction to acetylcholine blocking $\mathrm{K}^{+}$channels on the membrane are one and the same very variable process.

During long observation of spontaneously inactive neurons of the frontal cortex, in the overwhelming majority of cases ( 8 out of 10), a transition to a stable level of firing was detected. Silent neurons of the sensorimotor cortex, registered under the same conditions, as a rule remained inactive for a long time. Of the 17 inactive neurons of the sensorimotor cortex, spontaneous firing appeared only 
in 5 ones. Figure 2 II shows a neuron of the sensorimotor cortex, spontaneous activity of which did not occur during a 30-minute observation, but the reaction to acetylcholine gradually developed (Figure 2(A), II, 1 - 3). The response to glutamate remained unchanged, but the activation after-discharge increased (Figure 2(B), II, 1 - 3) similar to it occurred in the neuron of the frontal cortex during the formation of spontaneous activity (Figure 2(B), II, 1 - 3).

\subsection{Features of Responses to Glutamate Application in Neurons of the Sensorimotor and Frontal Cortex}

All the neurons tested in the layer $\mathrm{V}$ of both cortical regions responded to glutamate application by firing increase regardless of the level of their spontaneous activity. The response to glutamate applied to the soma from a channel coupled with the recording electrode was characterized by rapid development and short duration, with an activation after-discharge not exceeding 2500 ms (Figure $3(\mathrm{~A})$ ). Figure $3(\mathrm{~B})$ shows the statistical parameters of activation reactions to glutamate in neurons of the sensorimotor and frontal cortex. The distributions in Figure 3(B), I show that neurons of the frontal cortex respond to glutamate with a significantly shorter latency than neurons of the sensorimotor cortex $\left(\chi^{2}=\right.$ 12.53; $\alpha=0.006)$.

The same frontal neurons in reactions to glutamate achieve more powerful intensity. Most of the neurons of the frontal cortex (60\%) increased firing above the background level by $4-6 \mathrm{imp} / 200 \mathrm{~ms}$ (i.e., by $20-30 \mathrm{imp} / \mathrm{s}$ ). The neurons of the sensorimotor cortex to the glutamate application in most cases (68\%) formed weaker responses ( 2 - 4 pulses/200ms; i.e., 10 - 20 pulses/s), as shown in the distributions on Figure 3(B), III ( $\left.\chi^{2}=42.17 ; \alpha<0.00001\right)$. Maximum response value to glutamate remained constant for the neuronal groups with different level of spontaneous activity $(0-0 ; 0-4 ; 4-8 \mathrm{imp} / \mathrm{s}): 10-20 \mathrm{imp} / \mathrm{s}$ above the background activity for the neurons of sensorimotor cortex and $20-30 \mathrm{imp} / \mathrm{s}$ for the neurons of frontal cortex, as it was obtained for all neurons tested by glutamate (Figure 3(B), III). Only in the neuronal group with high-frequency spontaneous activity (above $8 \mathrm{imp} / \mathrm{s}$ ) the response intensity to glutamate in both cortical zones was characterized by a lower frequency. The range of firing response to glutamate application was from 1 to $11 \mathrm{imp} / 200 \mathrm{~ms}$ (5 - $55 \mathrm{imp} / \mathrm{s}$ ) above the background and was the same for the sensorimotor and frontal cortex (Figure 3(B), III). Consequently, the reactions to glutamate estimated by latency periods and firing intensity do not depend on spontaneous activity and are determined mainly by the input characteristics of the cell somata - the points of glutamate application. The input parameters of different neurons are different, but not varying when testing the same neuron in different conditions (Figure 2).

The most important difference between the sensorimotor and frontal cortical neurons in terms of the parameters of impulse responses to glutamate was the post-activation after-discharg. In Figure 3(B), II it is clearly seen that in $40 \%$ of neurons of the sensorimotor cortex, there is no activation aftereffect in responses to glutamate, and the most long-lasting after-discharges (up to $2500 \mathrm{~ms}$ 

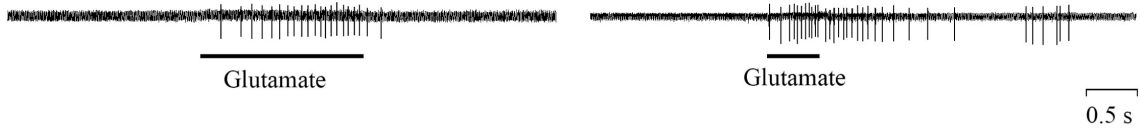

(A)
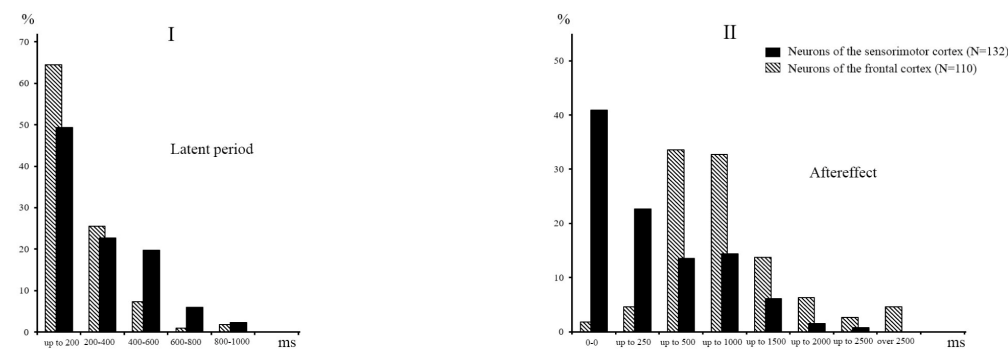

III

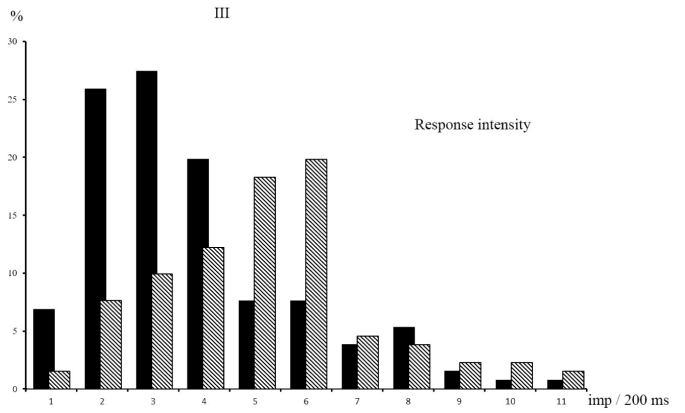

(B)

Figure 3. Comparative effect of glutamate application to neurons of the sensorimotor and frontal cortex. (A): examples of responses of a sensorimotor and a frontal cortical neurons to microiontophoretic application of glutamate: phoretic current, $80 \mathrm{nA}$ (negative pole inside the electrode). The duration of the phoretic current is indicated by a bar under each recording; (B): parameters of exciting responses to glutamate in neurons of the sensorimotor and frontal cortex: I: the distributions of neurons according to the latent periods of responses to glutamate, II: distributions of neurons according to the duration of activation after-discharge. III: distribution of neurons according to the intensity of the spike response above the background level (imp/200ms). The top right shows the number of tested neurons in the sensorimotor and frontal cortex.

or more) were found in responses of neurons in the frontal cortex $\left(\chi^{2}=86.80 ; \alpha\right.$ $<0.00001)$. The difference in this parameter indicates a weak expression of postexcitatory hyperpolarization in neurons of the frontal cortex compared to the sensorimotor one.

\subsection{Features of Responses to the Application of Acetylcholine in Neurons of the Sensorimotor and Frontal Cortex}

The responses of cortical neurons to the acetylcholine applications to their cell bodies were significantly different from the effects of glutamate, despite the fact that both transmitters cause an increase in impulse activity. Reactions to acetylcholine differed from glutamatergic excitation primarily in the dynamics of activation. An increase in the impulse frequency arose, as a rule, already after the 
cessation of the application of acetylcholine and required up to 10 seconds for development in both cortical regions. The duration of activation caused by acetylcholine was very long (Figure 2(A), I, 2 - 3; Figure 4(B)), which extended for several tens of seconds (with tendency for frontal neurons to respond more longer than $30 \mathrm{~s}$ ). This type of response to acetylcholine coincides with M-cholinergic excitation [17] [24].

The action of acetylcholine often did not lead to an increase in spike activity (Figure 2(A), I, 1 and II, 1; Figure 4(A), I, a and II, a). Of the 268 neurons tested in both cortical regions, 117 did not respond to acetylcholine, mainly

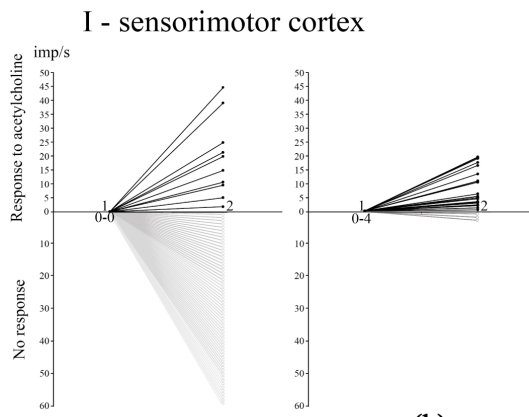

(a)

(b)

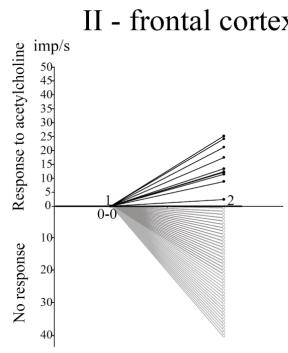

(a)

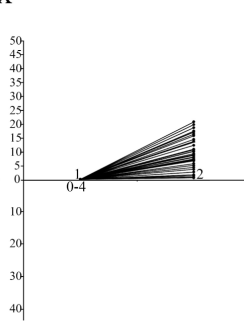

(b)

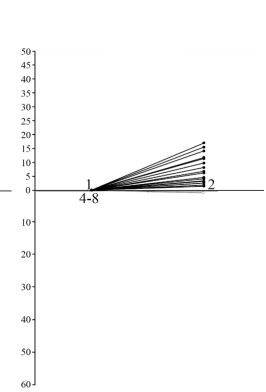

(c)

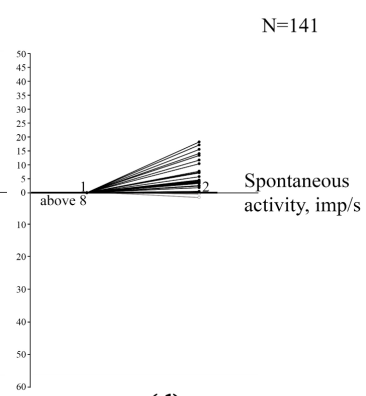

(d)

$\mathrm{N}=118$

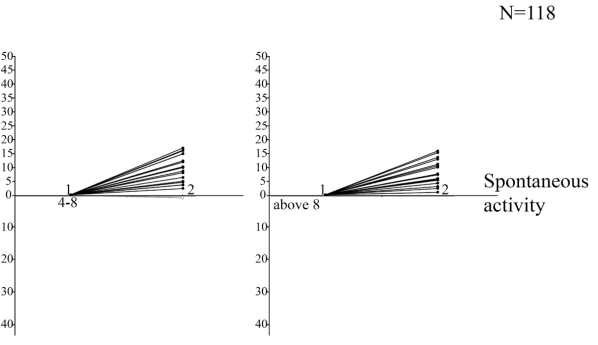

(c)

(d)

(A)

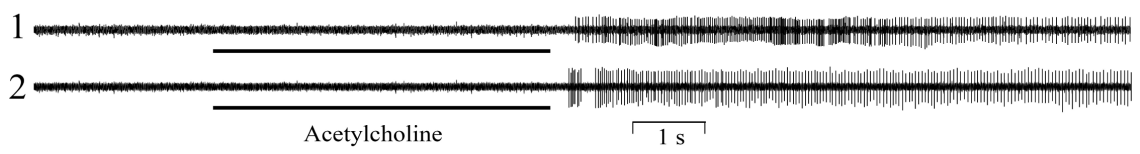

(B)

Figure 4. Comparative effect of the application of acetylcholine to the neurons of the sensorimotor and frontal cortex depending on the level of spontaneous activity. (A): Diagrams representing the reactions of all neurons of the sensorimotor cortex $(n=141)$ : I and the frontal cortex ( $\mathrm{n}=118)$ : II, grouped by the level of spontaneous activity ( $(\mathrm{a}),(\mathrm{b})$, (c), (d)), on the iontophoretic application of acetylcholine. The upper part of each diagram ((a), (b), (c), (d)) shows the increment of spike in responses to acetylcholine over the corresponding level of spontaneous activity. The level of spontaneous activity is indicated by point 1 on the abscissa axis for each diagram (imp/s). The spike increments caused by acetylcholine (ordinate axis, imp/s) are shown above point 2 on the abscissa axis. The lower part of each diagram ((a), (b), (c), (d)) shows the number of neurons that do not respond to acetylcholine for each level of spontaneous activity (points 1 and 2 on the abscissa axis); (B): Maximum spike reactions to application of acetylcholine in spontaneously inactive neurons of the sensorimotor (1) and frontal (2) cortex. The duration of the acetylcholine phoretic current is indicated by a bar under each recording. 
among spontaneously inactive cells, of which $85 \%$ were in the sensorimotor cortex and $78.4 \%$ in the frontal cortex. Spontaneously active neurons in the most cases reacted to acetylcholine. Their responses in each spontaneous firing range $(0-4 ; 4-8$; above $8 \mathrm{imp} / \mathrm{s})$, as well as the reactions of spontaneously inactive neurons, were evenly distributed from the lowest increments above the background level (up to $1 \mathrm{imp} / \mathrm{s}$ ) to the maximum possible. The maximum increment of impulse in response to acetylcholine was the largest among spontaneously inactive neurons and achieved $44.0 \mathrm{imp} / \mathrm{s}$ for cells of the sensorimotor cortex, and $25.3 \mathrm{imp} / \mathrm{s}$ for the frontal cortex (Figure 4(A), Ia and IIa; Figure 4(B); Table 1). With an increase in the initial frequency of background activity in nerve cells, a decrease in the maximum response to acetylcholine was observed: for the range $0-4 \mathrm{imp} / \mathrm{s}-20$ and $21 \mathrm{imp} / \mathrm{s}$ (correspondingly for neurons of the sensorimotor and frontal cortex), for higher frequency ranges this indicator dropped to $16.3 \mathrm{imp} / \mathrm{s}$ (Figure 4(A), I ((b)-(d)) and Figure 4(A), II ((b)-(d)); Table 1).

Thus, neurons that have different levels of spontaneous activity are grouped according to the degree of increase of those functional properties that determine their responses to acetylcholine. In this sense, spontaneously inactive cells represent a very heterogeneous group, while neurons that are active in the background, although they show a diversity in responses to acetylcholine, demonstrate a much narrower and almost the same range in the sensorimotor and frontal cortex (Figure 4(A)).

\section{Discussion}

\subsection{Features of Glutamatergic and Cholinergic Activation}

The difference in the activation spike reactions that occur in neurons by local exposure to glutamate and acetylcholine was noticed as far back as the middle of the last century. Almost all tested neurons were sensitive to glutamate [17] [18]. Their responses to the iontophoretic application of the transmitter had very short latent periods (from several ms to $1 \mathrm{~s}$ ), a very short exciting after-effect of about $1 \mathrm{~s}$ and were accompanied by a decrease in membrane resistance [19]; the resulting depolarization had a reversal potential of $0,-20 \mathrm{mV}$ [17]. The spike response to glutamate was highly stable: it remained constant when the transmitter was applied to the soma for many tens of presentations [20], did not change with decreasing temperature [21], under the influence of anesthesia [17]

Table 1. Maximum increments of firing responses to microiontophoretic applications of acetylcholine among neurons of sensorimotor and frontal cortex in different spontaneous frequency range.

\begin{tabular}{ccccc}
\hline Spontaneous activity range (imp/s) & $0-0$ & $0-4$ & $4-8$ & above 8 \\
\hline Maximum increment in sensorimotor cortex (imp/s) & 44.0 & 20.0 & 17.3 & 18.6 \\
Maximum increment in frontal cortex (imp/s) & 25.3 & 21.0 & 17.4 & 16.3 \\
\hline
\end{tabular}

The maximum increment was found among 141 sensorimotor cortical neurons and 118 frontal cortical neurons. 
[22] and metabolic inhibitors [23]. These data indicate that the ionic mechanism of glutamatergic depolarization is associated with an increase in $\mathrm{Na}^{+}$and $\mathrm{K}^{+}$ permeability [17] [19], and the only obstacle in the way of an activation spike response to glutamate is an impairment of the nonequilibrium distribution of these ions on both sides of the membrane.

The described mechanisms of glutamate action completely coincide with the effect of its application obtained in this study. All the neurons of the sensorimotor and frontal cortex, as in other areas of the brain, formed a rapid increase in spike activity in response to its iontophoretic application (Figure 2(B); Figure $3(\mathrm{~A})$ ). At the same time, analysis of the spike response parameters to glutamate revealed differences between the neurons of different cortical regions. The responses of nerve cells of the frontal cortex were of shorter latency and more powerful (Figure 3(B), I and Figure 3(B), III). A more significant activation after-discharge, registered to glutamate application in neurons of the frontal cortex (Figure 3(B), II) can serve as an explanation for these differences. A prolonged aftereffect is a consequence of the less pronounced post-excitatory hyperpolarization. This effect, which occurrence is associated with the development of the output $\mathrm{K}^{+}$current, can be weakened on the all over the entire structure only due to the lower density of $\mathrm{K}^{+}$channels on the neurons of the frontal cortex. In this connection, the membrane resistance rises, and then it contributes to the development of a faster and more powerful depolarization in response to glutamate applied to neuronal somata of the frontal cortex.

The difference in the density of $\mathrm{K}^{+}$channels on the membranes of neurons of the frontal and sensorimotor cortex is directly related to the responses to acetylcholine, since through interaction with M-cholinergic receptors, acetylcholine blocks the $\mathrm{K}^{+}$channels of cell membranes, increases membrane resistance and forms additional spike discharges after any depolarizing somatic effect [12] [13].

Blocking of $\mathrm{K}^{+}$channels was found by the reversal potential of cholinergic excitation, which was about $-100 \mathrm{mV}$, and by the increase in membrane resistance [12] [13] [17]. The spike response to the iontophoretic application of acetylcholine had a long latent period (from $1-2$ to 20 - $30 \mathrm{~s}$ ) and always outlasted the application by some tens of seconds [24]. Besides, acetylcholine during its iontophoretic injection often did not cause a spike reaction at all, and the effect itself turned out to be very variable, dependent on temperature, anesthesia and was blocked by an attenuation of energy metabolism [16] [17] [22] [23]. Thus, according to the formation mechanism (Figure 2) and physiological properties, the long-term reaction to acetylcholine was different from the rapid depolarization caused by glutamate.

Although many authors considered the rise in membrane resistance as a reason for a possible increase in the amplitude of depolarization that may occur simultaneously with the action of acetylcholine, and the decrease in post-excitatory hyperpolarization as a prolongating factor, the short duration and weak expression of these effects could not explain what is the mechanism of a long spike reaction to acetylcholine. One could pay attention to the fact that the action of 
acetylcholine led to an increase in the frequency and amplitude of miniature EPSPs, which formed a stationary synaptic flow in the soma [13]. But the origin of this stream remained unclear. It was further discovered that the effect of acetylcholine does not depend on the point (somatic or dendritic) of its application to the membrane of the neuron [25], and immediately after cholinergic exposure, the short-term application of glutamate to dendritic loci causes more short latent and more powerful spike reactions in the soma, than before exposure to acetylcholine [25]. Therefore, acetylcholine, closing $\mathrm{K}^{+}$channels on almost the entire membrane surface, increases the efficiency of dendrosomatic conductance in accordance with the W. Rall cable theory [10]. This mechanism of acetylcholine action at relatively small (about 10\%) [26] growth of membrane resistance leads to a very significant enhancement of amplitude characteristics of dendritic synaptic stream which is formed by numerous glutamatergic contacts on the dendrites. And if passing on the way of dendritic membrane EPSP amplitude can be reduced by tens of times [27], it allows acetylcholine to raise it tens times higher (Figure 4(B)). The different density of $\mathrm{K}^{+}$channels on the membrane of different neurons and in different structures (Figure 3(B), II) introduces additional diversity into the process of dendritic propagation regulated by acetylcholine.

\subsection{Heterogeneity of Neuronal Properties and Its Significance}

Structural and functional differences of neurons even within the same brain structure allow to consider the variability of neuronal properties as one of the basic principles of the nervous system construction [28]. A gradual change in the functional characteristics of pyramidal neurons in any hippocampal field is associated with genetic diversity and the different directions of the established neuronal connections [28]. Cell layers in the primary visual cortex consist of neurons with different types of dendritic trees, different levels of spontaneous activity and different peculiarities in responses to the light stimulation [29]. Within the layer $\mathrm{V}$ of the sensorimotor cortex, various changes in the spontaneous activity of various neurons were detected in thermobiological experiments during unidirectional changes of the medium temperature [16].

In the distributions shown in Figure 1 for neurons of the layer $\mathrm{V}$ of the sensorimotor and frontal cortex, it is clearly seen that cells with a various spontaneous activity do not distribute uniformly and neurons without background firing dominate among nerve cells. This means that there are exactly those neurons that have a genetically determined advantage over the others. Judging by the fact that spontaneously inactive neurons during the iontophoretic application of acetylcholine create the most powerful spike response sequences (Figure 4(A), Ia, IIa; Table 1), we can assume that this property is the most available for the formation of adaptive function. Warm-blooded animals are characterized by the additional incorporation of $\mathrm{K}^{+}$channels into the neuronal membranes [11], meanwhile the rate of the $\mathrm{M}$-cholinergic reaction significantly increases above $36^{\circ} \mathrm{C}$ [16]. As a result, a large number of spontaneously inactive neurons are formed 
(because of high density of $\mathrm{K}^{+}$channels) and prerequisites are created for regulation in a wide range of dendritic synaptic flow (both because of high density of $\mathrm{K}^{+}$channels and high rate of cholinergic reaction). At the same time, the lower density of $\mathrm{K}^{+}$channels on the membranes of the frontal neurons, tested by the long activation aftereffect in responses to glutamate (Figure 3(A) and Figure 3(B), II), explains the lower representation of spontaneously inactive neurons in the frontal cortex (Figure 1), their light transfer to the firing state (Figure 2, I) and a smaller value of the maximum spike increment in response to acetylcholine application(Figure 4(B); Table 1).

Particular attention should be paid to the fact that in spontaneously inactive neurons, acetylcholine rarely causes spike responses, despite the significant increase in spike reactions in some cases (Figure 4(A), Ia and IIa) In the sensorimotor cortex among spontaneously inactive neurons $85 \%$ did not respond to iontophoretic application of acetylcholine, in the frontal cortex $78 \%$ of spontaneously inactive neurons also did not respond to acetylcholine. In all likelihood, the fact of the absence of reactions to acetylcholine in most "silent" neurons is associated with the temperature of the incubating medium at which experiments were conducted $\left(32^{\circ} \mathrm{C}-34^{\circ} \mathrm{C}\right)$ and a very high density of $\mathrm{K}^{+}$channels on the membranes of neurons that did not respond to acetylcholine. Only the temperature-dependent increase in the rate of the cholinergic reaction that occurs at $\mathrm{t}=$ $36^{\circ} \mathrm{C}[16]$ can reduce the number of neurons that do not respond to acetylcholine at $32^{\circ} \mathrm{C}-34^{\circ} \mathrm{C}$ by $60 \%$. [16]. In Figure 5, taking into account the presented data and those obtained earlier [16], for a population of spontaneously inactive neurons of the sensorimotor cortex a hypothetical transition of responses to acetylcholine demonstrated with temperature lifting from $32^{\circ} \mathrm{C}-34^{\circ} \mathrm{C}$ to $35^{\circ} \mathrm{C}$ $37^{\circ} \mathrm{C}$. The number of neurons that did not respond to acetylcholine at $32^{\circ} \mathrm{C}$ $34^{\circ} \mathrm{C}$ becomes $34^{\circ} \mathrm{C}$ less at $35^{\circ} \mathrm{C}-37^{\circ} \mathrm{C}$ and the contribution of each neuron responded to acetylcholine increases with temperature lifting. Therefore, the functional properties of spontaneously inactive neurons of the sensorimotor cortex are significantly different from each other, which creates great opportunities for wide regulating spike activity, especially in the regime of warm-blooded.

In contrast to this group of neurons, the spontaneously active cells in response to acetylcholine not only express less maximum spike increments (Figure 4(A), I, b-d), but also exerted little changes with increasing temperature [16].

Similar differences between inactive and active neurons in reactions to acetylcholine were also observed in cells of the frontal cortex, but their spike responses were regulated in a smaller range (Figure 4(A), II and B, 2). Apparently, this circumstance is associated with features of functional control, which is carried out by cells of the studied cortical zones. And if the axons of neurons of the $\mathrm{V}$ layer of the motor cortex descend to the motor nuclei of the medulla oblongata, providing the late phase of the preparatory stage and the motor command [30], the frontal cortex regulates oculomotor activity, monitors visceral functions and emotional states [31] [32]. 


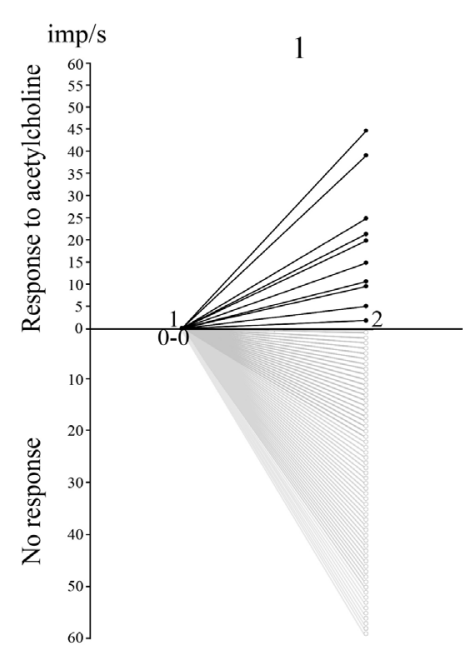

$32^{\circ} \mathrm{C}-34^{\circ} \mathrm{C}$

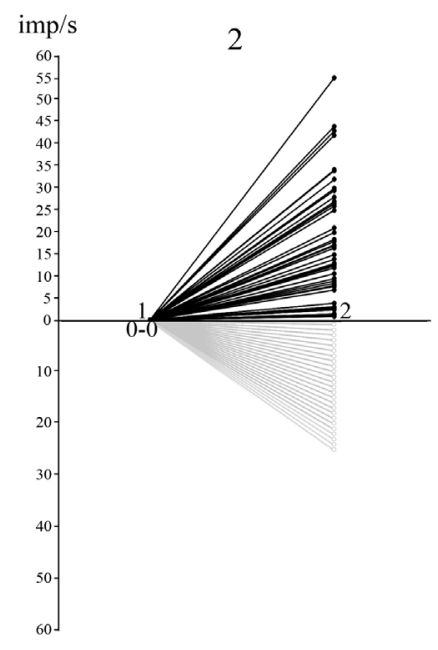

$35^{\circ} \mathrm{C}-37^{\circ} \mathrm{C}$

(A)

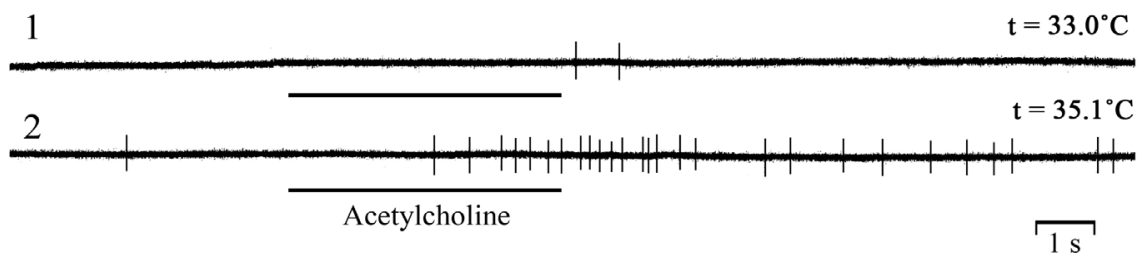

(B)

Figure 5. Hypothetical change in spike responses to application of acetylcholine with increasing temperature in spontaneously inactive neurons of the sensorimotor cortex. (A): Diagrams representing the reactions of spontaneously inactive neurons of the sensorimotor cortex $(n=69)$ to application of acetylcholine at $\mathrm{t}=32^{\circ} \mathrm{C}-34^{\circ} \mathrm{C}(1)$ and at $\mathrm{t}=$ $35^{\circ} \mathrm{C}-37^{\circ} \mathrm{C}(2): 1$ : the diagram completely corresponds to that shown in Figure 4, A, I, a; 2: changes in diagram 1 associated with an increase in temperature according to previous work [16]. Notation as in Figure 4; (B): Changes in the reaction to application of acetylcholine in a spontaneously inactive neuron of the sensorimotor cortex with an increase in temperature from $33.0^{\circ} \mathrm{C}(1)$ to $35.1^{\circ} \mathrm{C}$. Notation as in Figure 4 .

The effect of acetylcholine on the stationary synaptic flow in dendrites, provided that in each neuron the parameters involved in its formation are different, makes it possible to create a variety of impulse sequences in any specific brain structure responsible for the specific brain function. The greater the heterogeneity of the parameters affecting the effectiveness of dendrosomatic propagation, the higher the variety of possibilities of any area of the brain and the nervous system as a whole.

\section{Conclusion}

The cellular composition of any brain structure is heterogeneous in functional properties, which determine the level of spontaneous activity of neurons. The diversity by this feature determines for each neuron a certain spike response under the influence of acetylcholine, forms a large number of different spike sequences and, therefore, encodes a set of potential possibilities of the structure as a whole. 


\section{Acknowledgements}

This research was supported by the Russian Academy of Sciences.

\section{Conflicts of Interest}

The authors declare that they have no conflict of interest.

\section{References}

[1] Bogdanov, A.V. and Galashina A.G. (2003) Functional Relationships of the Motor Cortex Neurons during Training. Nauka, Moscow. (in Russian)

[2] Henneman, E. (1957) Relation between Size of Neurons and Their Susceptibility to Discharge. Science, 126, 1345-1347. https://doi.org/10.1126/science.126.3287.1345

[3] Karpuk, N.N. and Vorobiev, V.V. (2004) The Role of the Electrophysiological Properties of Neurons in the Mechanisms of Grouping their Discharges in the Cerebral Cortex. Neuroscience and Behavioral Physiology, 34, 881-888. https://doi.org/10.1023/B:NEAB.0000042572.67704.f5

[4] Kawaguchi, Y. (1993) Grouping of Nonpyramidal and Pyramidal Cells with Specific Physiological and Morphological Characteristics in Rat Frontal Cortex. Journal of Neurophysiology, 69, 416-431. https://doi.org/10.1152/jn.1993.69.2.416

[5] Leao, R.M., Li., S., Doiron, B. and Tzounopoulos, T. (2012) Diverse Levels of an Inwardly Rectifying Potassium Conductance Generate Heterogeneous Neuronal Behavior in a Population of Dorsal Cochlear Nucleus Pyramidal Neurons. Journal of Neurophysiology, 107, 3008-3019. https://doi.org/10.1152/jn.00660.2011

[6] McCormick, D.A., Connors, B.W., Lighthall, J.W. and Prince, D.A. (1985) Comparative Electrophysiology of Pyramidal and Sparsely Spiny Stellate Neurons of the Neocortex. Journal of Neurophysiology, 54, 782-806.

https://doi.org/10.1152/jn.1985.54.4.782

[7] Altemus, K.L., Lavenex, P., Ishizuka, N. and Amaral, D.G. (2005) Morphological Characteristics and Electrophysiological Properties of CA1 Pyramidal Neurons in Macaque Monkeys. Journal of Neuroscience, 136, 741-756.

https://doi.org/10.1016/j.neuroscience.2005.07.001

[8] Zakharova, N.V., Voronkov, D.N., Khudoerkov, R.M., Pasikova, N.V. and Mednikova, Y.S. (2018) Glia-Neuron Interactions in the Sensory-Motor Cortex of Warm-Blooded Animals (Guinea Pigs and Ground Squirrels) with Different Habitat Conditions and the M-Cholinergic Reaction of the Brain. Biophysics, 63, 207-214. https://doi.org/10.1134/S0006350918020264

[9] Bastian J. and Nguyenkim J. (2001) Dendritic Modulation of Burst-Like Firing in Sensory Neurons. Journal of Neurophysiology, 85, 10-22.

https://doi.org/10.1152/jn.2001.85.1.10

[10] Rall, W., Burke, R.E., Holmes, W.R., Jack, B., Redman, S.J. and Segev, I. (1992) Matching Dendritic Neuron Models to Experimental Data. Physiological Reviews, 72, S159-S186. https://doi.org/10.1152/physrev.1992.72.suppl_4.S159

[11] Kang, J., Huguenard, J.R. and Price, D.A. (1996) Development of BK Channels in Neocortical Pyramidal Neurons. Journal of Neurophysiology, 76, 188-198. https://doi.org/10.1152/jn.1996.76.1.188

[12] Krnjević, K., Pumain, R. and Renaud, L. (1971) The Mechanism of Excitation by Acetylcholine in the Cerebral Cortex. The Journal of Physiology, 215, 247-268. https://doi.org/10.1113/jphysiol.1971.sp009467 
[13] McCormick, D.A. and Prince, D.A. (1986) Mechanisms of Action of Acetylcholine in the Guinea-Pig Cerebral Cortex in Vitro. The Journal of Physiology, 375, 169-194. https://doi.org/10.1113/jphysiol.1986.sp016112

[14] Mednikova, Y.S., Kopytova, F.V. and Zhadin, M.N. (2010) Spontaneous Activity of Cortical Neurons in Vitro and Its Regulation by Acetylcholine. Neuroscience and Behavioral Physiology, 40, 986-992. https://doi.org/10.1007/s11055-010-9357-6

[15] Pennartz C.M.A., De Jeu, M.T.G., Geurtsen, A.M.S., Sluiter, A.A. and Hermes, M.L.H.J. (1998) Electrophysiological and Morphological Heterogeneity of Neurons in Slices of Rat Suprachiasmatic Nucleus. The Journal of Physiology, 506, 775-793. https://doi.org/10.1111/j.1469-7793.1998.775bv.x

[16] Isakova, A.V. and Mednikova, Y.S. (2007) Comparative Roles of Acetylcholine and Noradrenaline in Controlling the Spontaneous Activity of Cortical Neurons. Neuroscience and Behavioral Physiology, 37, 689-696. https://doi.org/10.1007/s11055-007-0069-5

[17] Krnjević, K. (1974) Chemical Nature of Synaptic Transmission in Vertebrates. Physiological Reviews, 54, 418-540. https://doi.org/10.1152/physrev.1974.54.2.418

[18] Krnjević, K. and Phillis, J.W. (1963) Iontophoretic Studies of Neurons in the Mammalian Cerebral Cortex. The Journal of Physiology, 165, 274-304. https://doi.org/10.1113/jphysiol.1963.sp007057

[19] Hori, N., Galeno, T. and Carpenter, D.O. (1987) Responses of Pyriform Cortex Neurons to Excitatory Amino Acids: Voltage Dependence, Conductance Changes, and Effects of Divalent Cations. Cellular and Molecular Neurobiology, 7, 73-90. https://doi.org/10.1007/BF00734991

[20] Mednikova, Y.S., Khokhlova, V.N. and Koshtoyants, O.K. (2013) Glutamatergic Nature of Spike Responses of Cortical Neurons to Sensory Stimulation. Sensory Systems, 27, 259-270. (in Russian)

[21] Mednikova, Y.S., Pasikova, N.V. and Kopytova, F.V. (2004) Effects of Temperature on Spike Activity of Cortical Neurons in Guinea Pigs. Neuroscience and Behavioral Physiology, 34, 459-465. https://doi.org/10.1023/b:neab.0000022630.53594.99

[22] Mednikova, Y.S., Kozlov, M.K. and Makarenko, A.N. (2019) Energy Aspects of Sodium Thiopental Action on Nervous Activity. Journal of Behavioral and Brain Science, 9, 33-53. https://doi.org/10.4236/jbbs.2019.92004

[23] Godfraind, J.M., Kawamura, H., Krnjević, K. and Pumain, R. (1971) Actions of Dinitrophenol and Some Other Metabolic Inhibitors on Cortical Neurons. The Journal of Physiology, 215, 199-222. https://doi.org/10.1113/jphysiol.1971.sp009465

[24] Krnjević, K. and Phillis, J.W. (1963) Acetylcholine-Sensitive Cells in the Cerebral Cortex. The Journal of Physiology, 166, 296-327. https://doi.org/10.1113/jphysiol.1963.sp007106

[25] Mednikova, Y.S., Karnup, S.V. and Loseva, E.V. (1998) Cholinergic Excitation of Dendrites in Neocortical Neurons. Journal of Neuroscience, 87, 783-796. https://doi.org/10.1016/s0306-4522(98)00166-3

[26] Ffrench-Mullen, J.M.H., Hori, N., Nakanishi, H., Slater, N.T. and Carpenter, D.O. (1983) Asymmetric Distribution of Acetylcholine Receptors and M Channels on Prepyriform Neurons. Cellular and Molecular Neurobiology, 3, 163-181. https://doi.org/10.1007/BF00735280

[27] Williams, S.R. and Stuart, G.J. (2002) Dependence of EPSP Efficacy on Synapse Location in Neocortical Pyramidal Neurons. Science, 295, 1907-1910. https://doi.org/10.1126/science.1067903 
[28] Cembrowski, M.S. and Spruston, N. (2019) Heterogeneity within Classical Cell Types in the Rule: Lessons from Pyramidal Neurons. Nature Reviews Neuroscience, 20, 193-204. https://doi.org/10.1038/s41583-019-0125-5

[29] Medini, P. (2011) Cell-Type-Specific Sub- and Suprathreshold Receptive Fields of Layer 4 and Layer 2/3 Pyramids in Rat Primary Visual Cortex. Journal of Neuroscience, 190, 112-126. https://doi.org/10.1016/j.neuroscience.2011.05.026

[30] Economo, M.N., Viswanathan, S., Tasic, B., Bas, E., Winnubst, J., Menon, V., Graybuck, L.T., Nguyen, T.N., Smith, K.A., Yao, Z., Wang, L., Gerfen, C.R., Chandrashekar, J., Zeng, H., Looger, L.L. and Svoboda, K. (2018) Distinct Descending Motor Cortex Pathways and Their Roles in Movement. Nature, 563, 79-84.

https://doi.org/10.1038/s41586-018-0642-9

[31] Simonov, P.V. (1987) The Motivated Brain. Nauka, Moscow. (In Russian)

[32] Vertes, R.P. (2006) Interactions among the Medial Prefrontal Cortex, Hippocampus and Midline Thalamus in Emotional and Cognitive Processing in the Rat. Journal of Neuroscience, 142, 1-20. https://doi.org/10.1016/j.neuroscience.2006.06.027 